\title{
Focal sparing of iron and fat in liver tissue in patients with hemosiderosis: diagnosis with combination of R2* relaxometry and proton density fat fraction calculation by MRI
}

\author{
Muşturay Karçaaltıncaba, İlkay İdilman, Azim Çelik
}

\begin{abstract}
PURPOSE
To demonstrate magnetic resonance imaging (MRI) findings of the focal sparing of iron and fat in liver tissue in patients with hepatic iron overload.

\section{MATERIALS AND METHODS}

We retrospectively reviewed 48 liver MRIs performed in patients with hemosiderosis from 2007-2009. We selected five $(10 \%)$ of these patients based on the observation of focal signal abnormalities in the posterior aspect of segment 4, anterior to the portal vein, on in- and out-of-phase T1-weighted gradient-echo images. To further characterize this signal abnormality in segment 4 , we calculated the simultaneous proton density fat fraction and R2* relaxometry using the investigational version of a quantitative chemical shift-based water-fat separation method known as IDEAL-IQ with a multiecho gradient echo sequence. Visual assessment and objective measurements were performed for the focal sparing of iron and fat.

\section{RESULTS}

The diagnoses of the five patients included thalassemia $(n=3)$, aplastic anemia $(n=1)$, and myelodosysplastic syndrome $(n=1)$. The focal sparing of iron was hypointense on R2* relaxometry compared to the rest of the liver. Fat fraction images failed to demonstrate a heterogeneous fat distribution. The focal sparing of fat in the liver with iron overload appeared as a reduced fat-containing area (fat fraction, $4 \%$ ) in segment 4 compared to the remainder of the left lobe (fat fraction, $12 \%$ ). The $\mathrm{R}^{*}$ map revealed no difference between the focal fat-sparing area and the rest of the liver. Areas in which focal fat and iron sparing occurred were not visible on post-contrast and diffusion-weighted images.

\section{CONCLUSION}

Focal fat and iron sparing in patients with liver siderosis can mimic a lesion. Quantitative MRI techniques can help to characterize abnormal signal changes in segment 4 of the liver in patients with hepatic iron overload and can eliminate the need for biopsy of pseudolesions.

Key words: • liver $\bullet$ magnetic resonance imaging $\bullet$ iron overload - hemosiderosis
\end{abstract}

From the Department of Radiology (M.K. $\bowtie$ musturayk@yahoo. com, I.I. ), Hacettepe University School of Medicine, Ankara, Turkey; and GE Healthcare (A.Ç.), MRI, Istanbul, Turkey.

Received 11 December 2010; revision requested 15 December 2010; revision received 15 December 2010; accepted 16 December 2010.

Published online 25 January 2011

DOI 10.4261/1305-3825.DIR.4134-10.1
$\mathbf{F}$ ocal fatty sparing and focal fatty infiltration can be observed in the posterior aspect of segment 4 due to aberrant venous drainage from gastric and pancreaticoduodenal veins and altered perfusion (1-6).

Hemosiderosis, most commonly seen in thalassemia patients, leads to an increased $\mathrm{R}^{*}$ value in the liver, which directly correlates with hepatic iron concentration (7). Recent advances in quantitative magnetic resonance imaging (MRI) methods have allowed for the separate and combined evaluation of liver fat and iron content (8-13). We used an investigative version of the chemical shift-based water-fat separation method, which addresses all known confounding factors, specifically those involving $\mathrm{T} 1, \mathrm{~T}^{*}$, spectral complexity of fat, noise bias, and eddy currents $(14,15)$.

We describe the MRI findings of focal iron and fat sparing, which can be seen as a focal signal abnormality in segment 4 on in-phase and outof-phase images in patients with hemosiderosis.

\section{Materials and methods \\ Selection of images and review of clinical data}

An institutional review board approved the study, and informed consent was obtained from all patients. We searched the radiology information system using the keywords "MRI", "liver" and "hemosiderosis" between 2007 and 2009. A total of 48 patients with hepatic iron overload who underwent liver MRI during this period were identified. Images were retrieved from picture archiving and communication system (PACS) and reviewed by two radiologists in consensus. Five patients had focal signal abnormalities in the posterior aspect of segment 4, anterior to the portal vein, on in-phase and out-of-phase T1-weighted images. Two of the patients died. The remaining three patients were evaluated by iterative decomposition of water and fat with Echo asymmetry and the Least Squares Estimation Quantification sequence (IDEAL-IQ), which allows quantification of liver fat and iron content to be performed simultaneously with $\mathrm{T}^{*}$ and field homogeneity corrections. We retrospectively reviewed patient records. Age, gender, pathologic diagnosis, and relevant medical history were documented.

\section{MRI technique \\ Imaging protocol}

Initial MRI examinations were performed on a 1.5 Tesla (T) MRI system (Achieva, Philips Medical Systems, Best, The Netherlands) using a four-channel phased-array body coil. The imaging protocol included the following sequences: coronal TRUE-FISP (repetition time/echo time [TR/TE], 3/1.5 ms; flip angle, $80^{\circ}$; slice thickness, $6 \mathrm{~mm}$; matrix, $256 \times 256$ ), axial T1-weighted gradient echo in-phase and out-of-phase (TR/TE, 117/4.6-2.3 ms; flip angle, $80^{\circ}$; slice thickness, $6 \mathrm{~mm}$; matrix, 


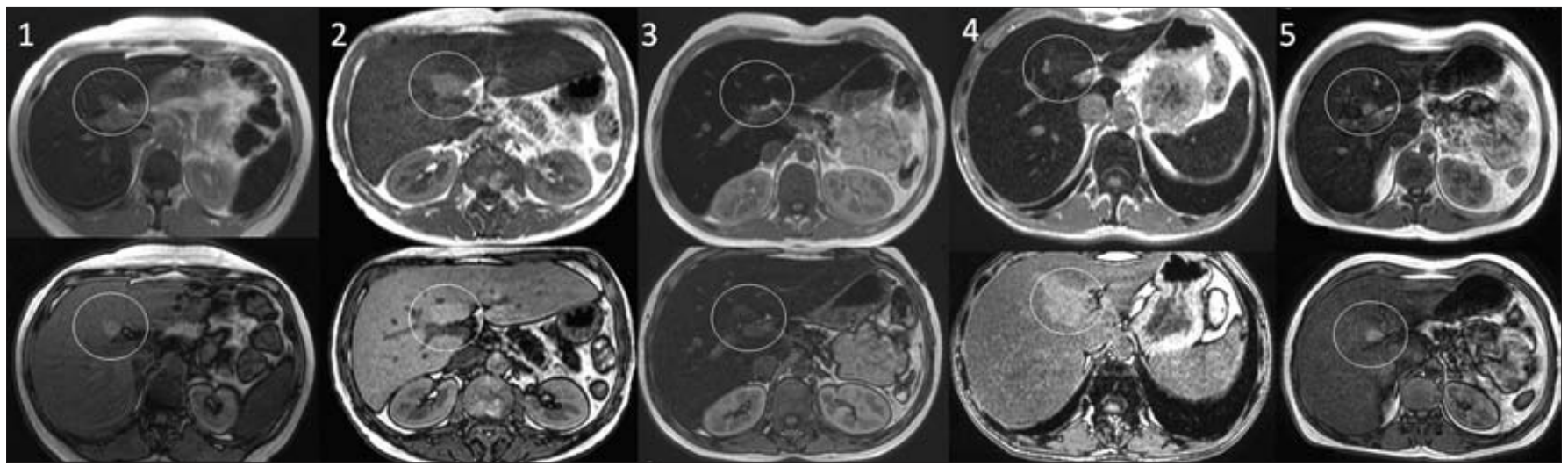

Figure 1. In-phase (top row) and out-of-phase (bottom row) T1-weighted MR images illustrate a focal signal abnormality (circle) at the posterior aspect of segment 4 in all five patients. In patients 1, 2 and 5, the signal abnormality appears to be the same on both images. In patients 3 and 4 , a signal drop is observed on the in-phase images.

256×256), axial heavily T2-weighted TSE (TR/TE, 416/120 ms; flip angle, $90^{\circ}$; slice thickness, $6 \mathrm{~mm}$; matrix, $512 \times 512)$, diffusion-weighted images $(b=0, b=50, b=600)$, axial breath hold three dimensional (3D) T1-weighted high resolution isotropic volume examination (THRIVE) (TR/TE, 3.9/1.9 ms; flip angle, $10^{\circ}$; reconstructed slice thickness, $2 \mathrm{~mm}$; matrix, 256×256) before intravenous contrast injection and at arterial, portal, and late venous phases after Gd-BOPTA injection (Multihance, Bracco, Milan, Italy) at a dose of $0.1 \mathrm{mmol} / \mathrm{kg}$. Gd-BOPTA injection was performed with a power injector (Medrad, Pittsburgh, Pennsylvania, USA) through a 20-gauge angiocath placed into the antecubital vein.

\section{Fat fraction and $R 2^{*}$ relaxometry calculations}

For R2* relaxometry and fat fraction calculations, MRI examinations were performed on a $1.5 \mathrm{~T}$ MRI system (GE Healthcare, Milwaukee, Wisconsin, USA) in June 2010. An eight-channel, phased-array body coil was used for the acquisition of this data. The MRI protocol included an in-phase and out-of-phase dual echo T1-weighted sequence and an investigational version of a quantitative chemical shift-based water-fat separation method known as IDEAL-IQ. The IDEAL-IQ sequence is used to simultaneously measure $\mathrm{R} 2$ * and fat fraction in the liver during a single acquisition scan. The IDEAL-IQ sequence takes $\mathrm{T}^{*}$ and field homogeneity variations into consideration for fat fraction calculations. The details of the IDEAL-IQ sequence have been described elsewhere (13). The parameters of the IDEAL-IQ sequence were TR, $12.9 \mathrm{~ms}$; FOV, 35-40 cm; matrix, $224 \times 160$; 125$\mathrm{kHz}$ bandwidth; and slice thickness, 5 $\mathrm{mm}$. A single 3D slab with 22-28 slices was acquired. We acquired six different echoes ranging from 1.6 to $9.8 \mathrm{~ms}$. The images were processed using the software provided by the manufacturer to create water, fat, in-phase, out-of-phase, $\mathrm{R} 2 *$, and fat fraction maps. All measurements were corrected for heterogene- ity using the homogeneity maps from auto-calibration.

\section{Results}

The diagnoses for the five patients included thalassemia $(n=3)$, aplastic anemia $(n=1)$, and myelodysplastic syndrome $(n=1)$ (Table 1). A focal signal abnormality in the posterior aspect of segment 4 appeared as a hyperintense area on out-of-phase images in all patients. In addition, signal drop was observed in two of five patients (patients 3 and 4) on in-phase images (Fig. 1). In the remaining three patients, there was no signal drop on the in-phase images.

Compared to the rest of the liver, focal iron sparing was hypointense on R2* relaxometry for patients 2 and 3 (Fig. 2). The R2* measurements from the area of focal iron sparing and the remainder of the liver in these patients were 93/199 s-1 and 201/309 $\mathrm{s}^{-1}$ (Table 2). Fat fraction images did not demonstrate heterogeneity in fat distribution or any evidence of fat sparing.

Table 1. Characteristics of focal signal abnormality in segment 4 on in-phase and out-of-phase T1-weighted, T2-weighted, and diffusionweighted MR images

\begin{tabular}{|c|c|c|c|c|c|}
\hline & Patient 1 & Patient 2 & Patient 3 & Patient 4 & Patient 5 \\
\hline In-phase T1-weighted & Hyperintense & Hyperintense & Isointense & Isointense & Hyperintense \\
\hline Out-of-phase T1-wieghted & Hyperintense & Hyperintense & Hyperintense & Hyperintense & Hyperintense \\
\hline $\begin{array}{l}\text { Signal drop on in-phase compared to } \\
\text { out-of-phase images }\end{array}$ & No & No & Yes & Yes & No \\
\hline Diffusion-weighted & Not visible & Not visible & Not visible & Not visible & Not visible \\
\hline
\end{tabular}



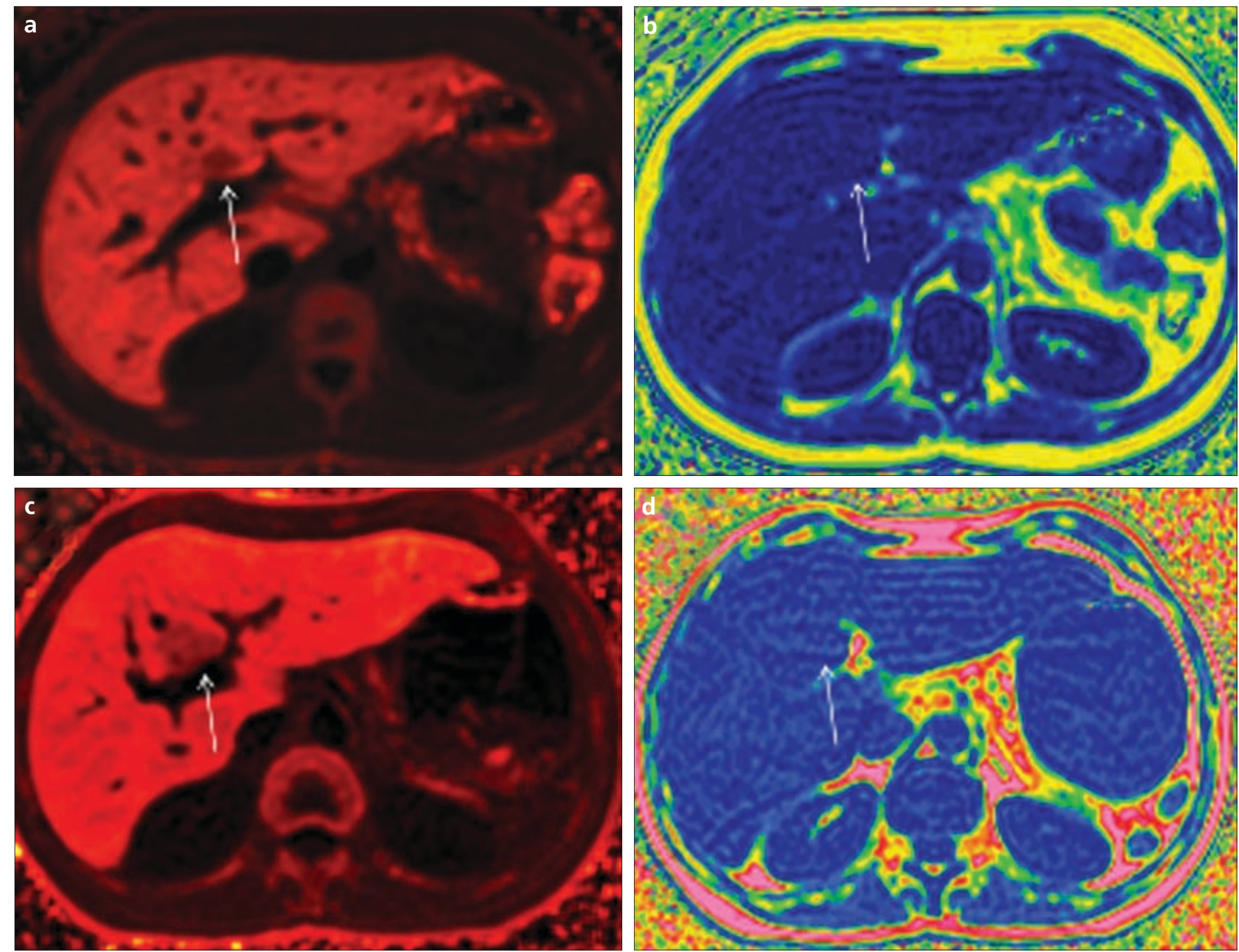

Figure 2. a-d. Focal iron sparing in patients 2 and 3 with hepatic iron overload. R2* map (a, c) and fat fraction images (b, d) of the liver show decreased focal signal (arrows) and homogeneous fat distribution, respectively, in segment 4 anterior to the portal vein.

Focal fat sparing in the liver with iron overload appeared as a reduced fat-containing focal area in segment 4 compared to the rest of the left lobe (Fig. 3). Upon visual assessment, the $\mathrm{R}^{*}$ map revealed no difference between the focal fat sparing area and the rest of the liver. $\mathrm{R}^{*}$ measurements from the area of focal iron sparing and the rest of the liver were 362 and $392 \mathrm{~s}^{-1}$, respectively. Correlative fat fraction values at these locations were $4 \%$ and $12 \%$. Areas with sparing of focal fat and iron did not demonstrate abnormal contrast enhancement on post-contrast images and were not visible on diffusion-weighted and T2weighted images.

\section{Follow-up}

Follow-up imaging was performed on all patients. The duration of follow- up ranged from 6-24 months. The size of the focal signal abnormality in segment 4 was stable in all patients.

\section{Discussion}

This study describes the diagnosis of focal iron and fat sparing in patients with hemosiderosis by using R2* map and fat fraction images in vivo. Focal areas with iron and fat sparing were initially diagnosed on in-phase and

Table 2. R2* map and fat fraction measurements of abnormal focal MRI signals in segment 4 and the left lobe of the liver in three patients with hemosiderosis

\begin{tabular}{lccc}
\hline Patients & Diagnosis & $\begin{array}{c}\text { Fat fraction } \\
\text { Segment 4 lesion/liver }\end{array}$ & $\begin{array}{c}\mathrm{R}^{*}\left(\mathrm{~s}^{-1}\right) \\
\text { Segment } 4 \text { lesion/liver }\end{array}$ \\
\hline Patient 2 & Focal iron sparing & $1 \% / 2 \%$ & $93 / 199 \mathrm{~s}^{-1}$ \\
Patient 3 & Focal iron sparing & $3 \% / 4 \%$ & $201 / 319 \mathrm{~s}^{-1}$ \\
Patient 5 & Focal fat sparing & $4 \% / 12 \%$ & $362 / 392 \mathrm{~s}^{-1}$
\end{tabular}



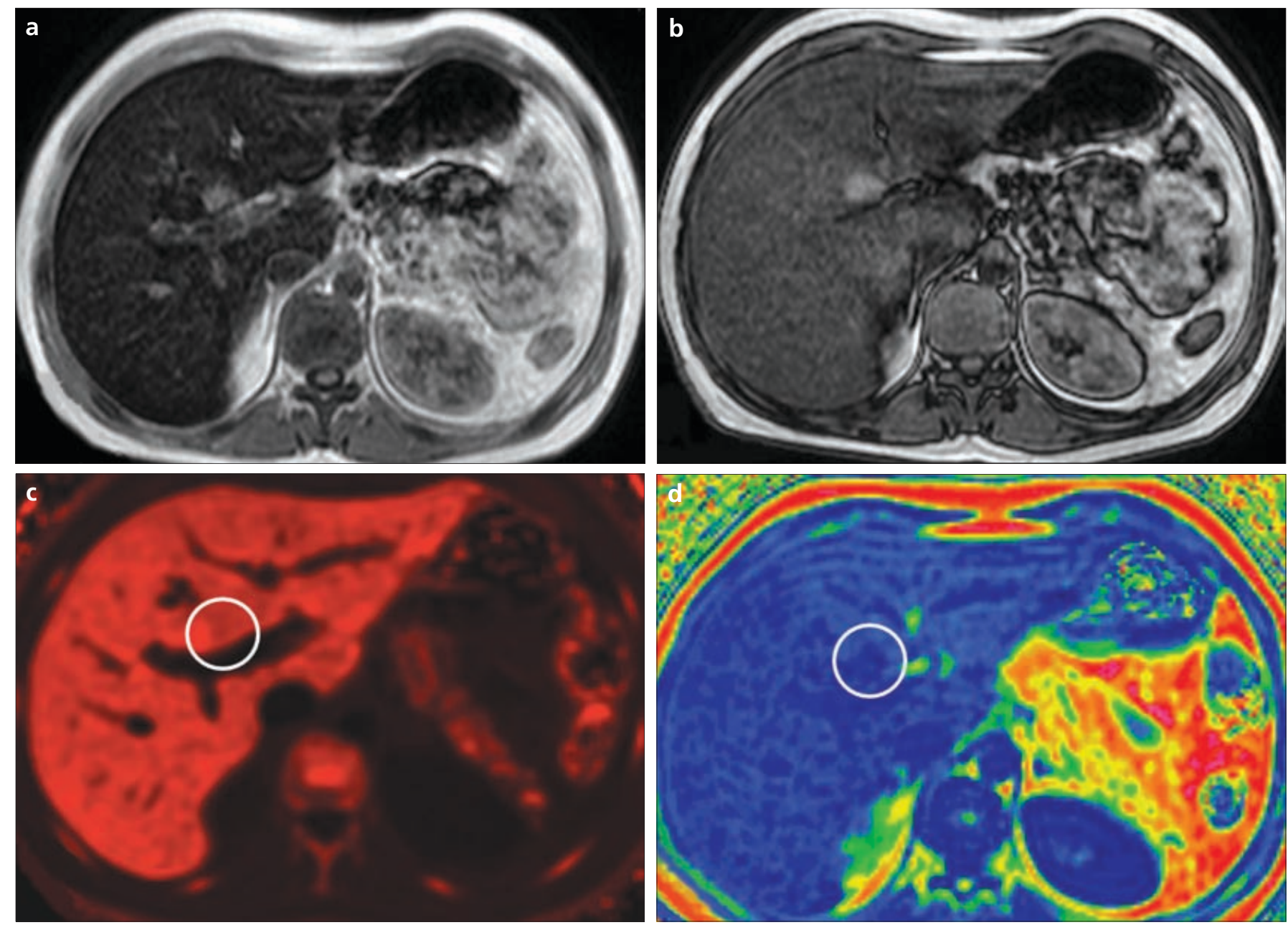

Figure 3. a-d. Focal fat sparing in patient 5 with hepatic iron overload. In-phase (a) and out-of-phase (b) images revealed a hyperintense signal abnormality in segment 4 . The corresponding $\mathrm{R} 2^{*} \operatorname{map}(\mathrm{c})$ was homogenous throughout the liver. Fat fraction images of the liver (d) revealed less fat in a focal area of segment 4 (circles) anterior to the portal vein, consistent with fat sparing. Note that fat content is higher in the rest of the left lobe compared to the right lobe.

out-of-phase T1-weighted images as focal signal changes when compared to diffuse iron-containing liver tissue in patients with hemosiderosis. The signal drop on in-phase images depends on the degree of steatosis and iron deposition; therefore, it is impossible to differentiate focal iron sparing from focal fat sparing. The IDEALIQ sequence allowed the characterization of spared focal areas based on differences in $\mathrm{R}^{*}$ values and fat fractions compared to the rest of the liver. Focal iron sparing appears to develop through a similar mechanism, leading to focal fatty infiltration and sparing. Focal fat sparing can be seen in other segments of the liver and can be nodular in shape (6). Therefore, focal fat or iron sparing at atypical locations can also be diagnosed by the combined calculation of $\mathrm{R} 2{ }^{*}$ and fat fraction.
Westphalen et al. demonstrated that iron overload limits the utility of inphase and out-of-phase images for steatosis detection, and $\mathrm{T} 2$ * correction is necessary for this assessment (16). Recently, Bydder et al. (8) and Boll et al. (9) reported the feasibility of calculating fat fraction in patients with hepatic iron deposition. Bydder et al. (8) injected a super paramagnetic iron oxide contrast agent in patients with steatosis and used low-flip angle multiecho gradient echo sequences, resulting in a slight overestimation of the fat fraction due to mono-exponential decay.

Initially, we believed that evaluating in-phase and out-of-phase images would allow the identification of patients with focal fat or iron sparing. In patients 2 and 5, the focal abnormal signal at the posterior aspect of segment 4 did not yield a signal drop on in-phase images. These patients, however, had focal iron sparing and focal fat sparing, respectively. The inphase images obtained for patient 3, who had focal iron sparing, revealed a signal drop. The signal drop on inphase images depends on the degree of steatosis and iron deposition and therefore cannot be used as an objective measurement when differentiating focal iron sparing from focal fat sparing. Theoretically, focal fat infiltration is also possible at this location. Focal fat infiltration can be identified by the combined calculation of the R2* map and liver fat fraction.

The limitations of this study include the lack of a biopsy correlation and the low number of patients. The lack of any change in the size of the abnormal signal at segment 4 , however, indicates the benign nature of this change. Focal fat sparing and focal iron sparing are 
relative terms; even in the absence of biopsy correlation, MRI can provide data on the relative quantities of fat and iron in these areas. Although the number of patients was low, this is, to our knowledge, the first study to describe the MRI characteristics of focal iron and fat sparing in patients with hepatic iron overload. This observation may allow for wider recognition of this type of signal change. The reliability of the method used for quantifying iron and fat in this study is debatable; however, this method works well at R2* values of up to $600 \mathrm{~ms}$ (unpublished observations).

Combination of liver fat and iron quantification techniques simultaneously from the same image dataset by IDEAL-IQ sequence can be used as a problem solving tool in patients with atypical focal signal changes and hemosiderosis. Focal iron and fat sparing in segment 4 of the liver can mimic a lesion and can be characterized by MRI in patients with hepatic iron overload. The findings of this study should facilitate the diagnosis of atypically localized sparing in this patient group.

\section{Conflict of interest disclosure}

The authors declared no conflicts of interest.

\section{References}

1. Matsui O, Takahashi S, Kadoya M, et al. Pseudolesion in segment IV of the liver at CT during arterial portography: correlation with aberrant gastric venous drainage. Radiology 1994; 193:31-35.

2. Matsui O, Kadoya M, Takahashi S, et al. Focal sparing of segment IV in fatty livers shown by sonography and CT: correlation with aberrant gastric venous drainage. AJR Am J Roentgenol 1995; 164:1137-1140.

3. Kawamori Y, Matsui O, Takahashi S, Kadoya M, Takashima T, Miyayama S. Focal hepatic fatty infiltration in the posterior edge of the medial segment associated with aberrant gastric venous drainage: CT, US, and MR findings. J Comput Assist Tomogr 1996; 20:356-359.

4. Fukukura $\mathrm{Y}$, Fujiyoshi $\mathrm{F}$, Inoue $\mathrm{H}$, et al. Focal fatty infiltration in the posterior aspect of hepatic segment IV: relationship to pancreaticoduodenal venous drainage. Am J Gastroenterol 2000; 95:3590-3595.

5. Yamagami $\mathrm{T}$, Takeuchi $\mathrm{Y}$, Inaba $\mathrm{Y}$, Matsueda K, Arai Y, Maeda T. Correlation of a defect of portal perfusion in the dorsal part of segment IV of the liver on CT arterial portography with inflow of the aberrant pancreaticoduodenal vein. Br J Radiol 1999; 72:552-555.

6. Karcaaltincaba M, Akhan O. Imaging of hepatic steatosis and fatty sparing. Eur J Radiol 2007; 61:33-43.

7. Wood JC, Enriquez C, Ghugre N, et al. MRI $\mathrm{R} 2$ and $\mathrm{R} 2$ * mapping accurately estimates hepatic iron concentration in transfusiondependent thalassemia and sickle cell disease patients. Blood 2005; 106:1460-1465

8. Bydder M, Shiehmorteza M, Yokoo T, et al. Assessment of liver fat quantification in the presence of iron. Magn Reson Imaging 2010; 28:767-776.
9. Boll DT, Marin D, Redmon GM, Zink SI, Merkle EM. Pilot study assessing differentiation of steatosis hepatis, hepatic iron overload, and combined disease using twopoint dixon MRI at $3 \mathrm{~T}$ : in vitro and in vivo results of a $2 \mathrm{D}$ decomposition technique. AJR Am J Roentgenol 2010; 194:964-971.

10. Taouli B, Ehman RL, Reeder SB. Advanced MRI methods for assessment of chronic liver disease. AJR Am J Roentgenol 2009; 193:14-27.

11. Cassidy FH, Yokoo T, Aganovic L, et al. Fatty liver disease: MR imaging techniques for the detection and quantification of liver steatosis. Radiographics 2009; 29:231-260.

12. Ma X, Holalkere NS, Kambadakone RA, Mino-Kenudson M, Hahn PF, Sahani DV. Imaging-based quantification of hepatic fat: methods and clinical applications. Radiographics 2009; 29:1253-1277.

13. Reeder SB, Robson PM, Yu H, et al. Quantification of hepatic steatosis with MRI: the effects of accurate fat spectral modeling. J Magn Reson Imaging 2009; 29:1332-1339.

14. Hines $\mathrm{CD}, \mathrm{Yu} \mathrm{H}$, Shimakawa A, McKenzie CA, Brittain JH, Reeder SB. T1 independent, T2* corrected MRI with accurate spectral modeling for quantification of fat: validation in a fat-water-SPIO phantom. J Magn Reson Imaging 2009; 30:12151222.

15. Yu H, Shimakawa A, McKenzie CA, Brodsky E, Brittain JH, Reeder SB. Multiecho water-fat separation and simultaneous R2* estimation with multifrequency fat spectrum modeling. Magn Reson Med 2008; 60:1122-1134.

16. Westphalen AC, Qayyum A, Yeh BM, et al. Liver fat: effect of hepatic iron deposition on evaluation with opposed-phase MR imaging. Radiology 2007; 242:450-455. 